\title{
Optimal strategies for the use of genomic selection in dairy cattle breeding programs
}

\author{
M. Wensch-Dorendorf, ${ }^{* 1}$ T. Yin, $\dagger^{1}$ H. H. Swalve, ${ }^{* 2}$ and S. König \\ *Institute of Agricultural and Nutritional Sciences, University of Halle, 06099 Halle, Germany \\ †Institute of Animal Breeding and Genetics, University of Göttingen, 37075 Göttingen, Germany \\ $\ddagger$ Department of Animal Breeding, University of Kassel, 37213 Witzenhausen, Germany
}

\section{ABSTRACT}

The objective of the present study was to conduct a stochastic simulation study on the possible benefits of an application of genomic selection in dairy cattle breeding programs according to a variety of selection schemes. In addition, the heritability of the trait in question, the accuracy of genomic breeding values, and the number of animals to be genotyped were varied. Specifically, the question of genotyping males, females, or both, was addressed. Selection schemes were compared with a young bull breeding program. The main criterion for comparison was the average of true breeding values of selected young males to be used as replacements for artificial insemination bulls. Stochastic simulations were run with 50 repetitions each to generate individuals with phenotypes, breeding values estimated by BLUP, and true breeding values. Genomic breeding values were generated from true breeding values with defined accuracy. Examined scenarios included a group of selection schemes that featured genotyping of parents of future bulls only. Such schemes can be viewed as improvements of young bull programs, and they were found to be competitive with or superior to a classical young bull program. However, a genomic breeding program usually involves at least genotyping young male candidates. A second group of selection schemes reflected this requirement. Scenarios in this group were found to be superior over the young bull program by 1.0 to 1.2 standard deviations of the average true breeding value of young male candidates. Within this group of scenarios, one scheme referred to an ideal situation under which genotypes for male calves were available without limitation. Using the average of true breeding values as the criterion for comparison, this idealistic scenario was competitive with other scenarios only if the reliability of genomic breeding values was larger than 0.50. Conventionally,

Received December 16, 2010.

Accepted April 5, 2011.

${ }^{1}$ Both authors contributed equally to this study.

${ }^{2}$ Corresponding author: hermann.swalve@landw.uni-halle.de not all males available will have genotypes, and the 2 most promising scenarios included a preselection step for dams of future bulls. This preselection step can be based on conventional BLUP estimated breeding values for bull dams, because differences with a scheme under which both parents and the resulting male offspring are genotyped were marginal. Genotyping of young male candidates should be the focus of activities of today's breeding organizations.

Key words: genomic selection, breeding program, preselection

\section{INTRODUCTION}

Basing selection decisions on the results of genotyping animals for high-density arrays of SNP denotes what is now called genomic selection. It consists of estimating breeding values by applying previous knowledge from so-called calibration or reference samples. This new tool in dairy cattle breeding has the potential of overcoming the existing obstacle of a costly and time-consuming progeny test for future bulls to be used in AI. Meuwissen et al. (2001) have shown the enormous potential of such a strategy, and Schaeffer (2006), using simple deterministic calculations, outlined the use of genomic selection comparing traditional and new strategies with respect to genetic gain and costs. Since then, numerous authors have studied the benefits of genomic selection in dairy cattle breeding programs.

It is obvious that the accuracy of a genomic evaluation $\left(\boldsymbol{r}_{m g}\right)$; that is, the correlation between a breeding value estimated from genomic data and incorporating the results from calibration samples with the true breeding value, plays a critical role in the success of implementing genomic selection into a breeding program. The current knowledge on what can be achieved considering this parameter indicates accuracies close to those obtained from costly and time-consuming progeny testing. Among others, Goddard and Hayes (2009) emphasized that the size of the calibration sample (which is composed of bulls with high accuracies of their progeny test genetic evaluations) is the most critical parameter determining $r_{m g}$. However, other factors, such as 
the extent of linkage disequilibrium in the specific population, will also play a role. VanRaden et al. (2009) showed that reliabilities $\left(r_{m g}^{2}\right)$ of genomic breeding values were as high as $69 \%$ for a calibration sample of 3,576 bulls but depended on the trait analyzed. Lower values will be found for traits with lower heritability. Hayes et al. (2009) also showed the dependencies between the size of the calibration sample and the reliability obtained. The latest results from various countries were presented at the Ninth World Congress on Genetics Applied to Livestock Production held in Leipzig, Germany, in 2010. Wiggans et al. (2010) reported a value for $r_{m g}^{2}$ of $71 \%$ for milk yield and $76 \%$ for fat yield, based on a calibration sample of 7,113 Holstein animals consisting mostly of bulls but also incorporating cows. For the German calibration sample of 5,025 bulls, Liu et al. (2010) presented values for $r_{m g}^{2}$ of $68 \%$ for milk yield and $72 \%$ for fat yield. Lund et al. (2010) showed results from the until-now largest reference population consisting of bulls that has been assembled for the Holstein breed by a team effort in France, the Netherlands, Denmark, Sweden, Finland, and Germany. This reference population included 15,966 bulls and has further increased the reliabilities obtained in the respective individual countries by substantial margins. For the German calibration sample, reliabilities based on the combined reference population and compared with the national reference were increased by $11 \%$, averaged over traits (Lund et al., 2010). On the other hand, countries with smaller breeds will adopt genomic selection and will have to cope with lower accuracy. Thus, from the wide range of results for reliabilities or accuracies of genomic breeding values that have been reported to date, it will be necessary to reflect this wide range in model calculations studying the benefits of genomic selection.

In a few countries or even through collaborative efforts across countries, procedures for genomic selection have already been implemented. Interbull (2010) lists 8 Holstein populations from 11 countries that provided information for the validation of their genomic evaluation system. However, within population or country, breeding organizations at present are facing the question of how to make use of the genomic evaluation provided and especially have to determine which animals should be genotyped as candidates for selection. In conventional progeny-testing programs, the most crucial step of selection is the selection of bull dams, because potential selection candidates are spread across herds, regions, and even countries and are subject to preferential treatment. The male paths of selection are less problematic as long as a progeny test with a suf- ficient number of progeny is conducted in an unbiased way. Therefore, a topic of intense discussion is whether to genotype males, females, or both sexes as candidates for selection.

The overall objective of the present study was to conduct a stochastic simulation study on the possible benefits of an application of genomic selection in dairy cattle breeding programs. A stochastic simulation was preferred over a deterministic calculation because means and variances of individuals can be studied and a stochastic simulation allows for greater flexibility when analyzing the simulated data. Within the general objective, the present study aimed to vary the most critical parameters and scenarios. This specifically pertains to genotyping a wide range of animals, females and males, and varying the reproductive rate of females, thus resulting in a categorization of general strategies and varying selection intensities within strategies. The overall approach was to take the view of a breeding organization. This is not only reflected by varying selection intensities but also by defining the main criterion for comparison as the average of true breeding values of the selected bulls that are the ultimate product of an AI organization. Additionally, the genetic structure and inbreeding coefficients of the bull calves selected to be future replacements for bulls used in widespread AI were examined.

\section{MATERIALS AND METHODS}

Simulation was based on the application of the QMSim program (Sargolzaei and Schenkel, 2009); QMSim is a very powerful simulation program to be used for the stochastic simulation of animal populations covering a population genetic level as well as the genomic level and featuring the simulation of historical populations to create linkage disequilibrium. The genomic level includes the capability of defining several chromosomes with QTL and marker maps. In the present study, however, QMSim was used to simulate populations consisting of individual animals under a defined genetic structure of the entire population along with their true breeding values and phenotypes. Features simulating high-density marker maps were not used because the objective of this study was not to simulate the entire process of genomic selection including calibration samples, estimation of genomic breeding values (GBV), validation, and finally the use of formulas to predict breeding values for young selection candidates without phenotypes. Rather, GBV; that is, breeding values estimated from genomic data without the use of phenotypes, were simulated with defined accuracies based on the true breeding values. 


\section{Simulation of Populations Using QMSim}

A founder population was generated over 1,000 generations with a constant population size of 50,500 animals, the last generation containing 50,000 females and 500 males. Based on this founder population, 20 generations of a population under selection were simulated. Animals of the last historic generation were parents of generation 1 for the population under selection. For females, a replacement rate of $25 \%$ was set. For males, $50 \%$ of the individuals were replaced by males from the following generation. Selection of males and females under these replacement strategies was based on BLUP EBV for a single trait. Within the selected fractions of males and females, mating was at random. The assumption of random mating appears to hold as breeders have many individual reasons for choosing a bull from those selected and offered by breeding organizations. In contrast, a strictly assortative mating scheme would be very unlike what happens in the real world of dairy cattle breeders. From generation 1 to generation 5, the number of females in the population was increased by $25 \%$ of the original population size to reflect the growth of a superior breed. Thus, the number of females for generations 1 to 5 was $50,000,62,500,75,000,87,500$, and 100,000 . The number of sires was kept at 500. After generation 5 , the size of the population was constant.

Within the simulation of generations 1 to 19, the reproductive rate of females was set such that 1 offspring, with a probability of $50 \%$ for being male or female, was produced by every cow. However, for potential bull dams, this may not be applicable. Modern dairy cattle breeding programs regularly use embryo transfer to increase the reproductive rate of females. In the simulation studies by Sorensen and Sorensen (2010), Buch et al. (2010), and Pedersen et al. (2010), the success rate of embryo transfer was set to 5 offspring per cow. Although such a value may be achievable, in this study we used a much lower success rate such that in the last generation 20, all cows produced 1 male offspring. In a second set of simulation scenarios, the rate of male offspring per bull dam in generation 20 was set to 0.5 .

In total, 2 single traits with mean zero and phenotypic SD of 1.00 were independently simulated in different simulation runs for a heritability of 0.10 and 0.30. Simulations using QMSim were repeated 50 times. Within each repetition, means for true breeding values for desired numbers of selected bull calves were computed. Then, means for repetitions were averaged and SD were calculated across repetitions and within scenario and number of selected bull calves.

Selection was based on BLUP EBV as supplied by QMSim. In summary, the variables simulated for every individual applying QMSim and used for further processing were phenotypes, conventional BLUP EBV, true breeding values (TBV), and the inbreeding coefficients. In all further steps, only animals from generation 20 were considered as young candidates for selection to mimic the actual situation of breeding organizations.

\section{Simulation of Genomic EBV}

Based on the output of the QMSim program, all further computational steps were undertaken by our own programming using SAS and C as programming languages. Given the objective to analyze various scenarios differing by the correlation between breeding values estimated from genomic data with the TBV, direct GBV were simulated based on the TBV as supplied by QMSIM according to the following formula:

$$
g b v_{i}=\left[\left(1-r_{m g}^{2}\right)^{1 / 2} z g_{i}+r_{m g} \cdot T B V_{i} / s d\left(T B V_{t}\right)\right] s d\left(T B V_{t}\right)
$$

where $g b v_{i}$ is the genomic breeding value of animal $i$, $r_{m g}$ is the accuracy of the genomic breeding value, $T B V_{i}$ is the true breeding value of animal $i, \operatorname{sd}\left(T B V_{t}\right)$ is the standard deviation of TBV in generation $t$, and $z g$ is a random variable sampled from $N(0,1)$.

For some scenarios, the population was divided into groups to imitate large herds to be used as a nucleus for selection activities. Herd sizes were from 200 to 800 cows; that is, with a mean of 500 cows and an SD of herd size of 100 . Selection of such herds by breeding organizations most likely will be according to phenotypic level. This was mimicked by deriving herd effects, which were correlated with the average genetic level of the herd, according to

$$
\begin{gathered}
h m_{j}= \\
{\left[\left(1-r_{h m . E B V}^{2}\right)^{1 / 2} z g_{j}+r_{h m . E B V} \cdot E B V_{H e r d j} / s d\left(E B V_{H e r d}\right)\right] s d_{h m},}
\end{gathered}
$$

where $h m_{j}$ denotes the herd effect of herd $j, r_{h m . E B V}$ is the correlation between the vector of herds effects $(\mathrm{hm})$ and the vector of herd EBV averages $\left(E B V_{H e r d}\right)$, and $\operatorname{sd}\left(E B V_{\text {Herd }}\right)$ is its standard deviation; $E B V_{\text {Herdj }}$ is the average EBV of all cows in herd $j$. A fixed value of 0.3 was used for $r_{h m . E B V}$.

\section{Scenarios for Comparison}

All scenarios centered on comparing the genetic merit of selected young bull calves as potential replacements for AI bulls. Figure 1 gives an overview of the scenarios simulated for comparisons. In Figure 1, scenarios are grouped by 3 main strategies. Scenario REF is the ref- 
erence scenario referring to the initial step of a conventional progeny-testing program; that is, the selection of young bulls for progeny testing. In this scenario, bull calves are selected through selection of parents with outstanding EBV; that is, by a pedigree index defined as the mean of parents' BLUP EBV. Selection simply consisted of selecting the best $n$ bull calves according to their pedigree index in a single step. Thus, scenario REF denotes a young bull program. In conventional programs, these young bulls would then be subject to progeny testing and their genetic merit with accuracy typical for progeny testing would only be known 5 yr later. Given that the conventional estimation of breeding values would be unbiased, selection among these calves according to pedigree index would result in average values for TBV corresponding to average EBV that would be available 5 yr later. However, REF differs from a conventional progeny-testing program because only the preselection step of a conventional program was applied for this scenario.

Scenario GPAR consists of a group of scenarios describing a situation in which a breeding organization is reluctant to use the technology of genomic selection or wishes to minimize any costs associated with it. In such a situation, a breeding organization could rely on the availability of GBV for bulls and especially sires of bulls already genotyped by other breeding organizations worldwide. Additionally, many potential bull dams will already have been genotyped, financed by the individual owner rather than the breeding organization. Such a scenario can also be viewed as a scenario describing the situation of a breeding organization not having access to genomic selection technology and hence relying on publicly available data or on data supplied by individual breeders. This could also denote the case of individual traders of genetic material that select bull calves based on genomic evaluations of parents, which will or may be publicly available and market these young bulls for AI or as natural service bulls under the term "genomically selected." In all GPAR scenarios, GBV are available for parents; that is, potential bull sires and bull dams. Scenario $\mathbf{G P A R}$ _ALL denotes a situation in which all females that could possibly be used as bull dams; that is, all females, will have a GBV, and the GBV of potential sires of bulls will be publicly available. In GPAR_ BD_BLUP, a preselection step is introduced such that bull dams with a high conventional EBV will be genotyped. Scenario GPAR_BD_PHEN is similar to scenario GPAR_BD_BLUP; however, bull dams are preselected according to phenotype rather than EBV. For GPAR_BD_BLUP and GPAR_BD_PHEN, the number of preselected females was varied to build selection pools of 50,100 , or 500 females. These numbers were chosen to reflect the current situation in which

\begin{tabular}{|c|c|c|c|c|c|}
\hline \multicolumn{6}{|c|}{ GPROG - Bull calves are genotyped } \\
\hline $\begin{array}{c}\text { ALL } \\
\text { All } \\
\text { bull calves are } \\
\text { genotyped }\end{array}$ & $\begin{array}{l}\text { RANDOM } \\
n \\
\text { bull calves are } \\
\text { genotyped at } \\
\text { random }\end{array}$ & $\begin{array}{l}\text { BD_BLUP } \\
\text { n bull dams } \\
\text { are } \\
\text { pre-selected } \\
\text { based on } \\
\text { BLUP-EBV, } \\
\text { their bull } \\
\text { calves are } \\
\text { genotyped }\end{array}$ & $\begin{array}{l}\text { BD_PHEN } \\
\text { n bull dams } \\
\text { are } \\
\text { pre-selected } \\
\text { based on } \\
\text { phenotype, } \\
\text { their bull } \\
\text { calves are } \\
\text { genotyped }\end{array}$ & $\begin{array}{l}\text { BD_HERD } \\
\text { n bull dams } \\
\text { are } \\
\text { pre-selected } \\
\text { from specific } \\
\text { herds, } \\
\text { their bull } \\
\text { calves are } \\
\text { genotyped }\end{array}$ & $\begin{array}{c}\text { BD_GENO } \\
n \\
\text { bull calves } \\
\text { are } \\
\text { pre-selected } \\
\text { on PI-GBV } \\
\text { and } \\
\text { genotyped }\end{array}$ \\
\hline \multicolumn{6}{|c|}{ GPAR - Parents are genotyped, bull calves are not } \\
\hline \multicolumn{2}{|c|}{$\begin{array}{l}\text { ALL } \\
\text { All parents are } \\
\text { genotyped; } \\
\text { No shortage of } \\
\text { bull dams with } \\
\text { genotypes }\end{array}$} & \multicolumn{2}{|c|}{$\begin{array}{l}\text { BD_BLUP } \\
\text { Pre-selection of } n \\
\text { bull dams based on } \\
\text { BLUP-EBV }\end{array}$} & \multicolumn{2}{|c|}{$\begin{array}{l}\text { BD_PHEN } \\
\text { Pre-selection of } n \\
\text { bull dams based on } \\
\text { phenotype }\end{array}$} \\
\hline \multicolumn{6}{|c|}{ REF - Bull calves are selected on pedigree index } \\
\hline
\end{tabular}

Figure 1. Characteristics of the simulated scenarios. PI-GBV $=$ genomic pedigree index, calculated as average genomic breeding value (GBV) of parents. 
the number of potential bull dams is still limited and can be viewed as a contrast to an unlimited access to genotyped bull dams simulated in GPAR_BD_ALL.

In all GPROG scenarios, bull calves as potential candidates for selection are always genotyped and thus have a GBV. Disregarding costs and all other expenses within a breeding program, such scenarios will always have the clear advantage of having a GBV associated with each bull, the final product of all efforts of the breeding organization. In GPROG_ALL, the "bull calf program" as suggested by König and Swalve (2009) is represented. All male calves will have a GBV, thus assuming an ideal situation in which costs of genotyping would be negligible. Scenario GPROG_RANDOM is a variation of GPROG_ALL but now defining limits to several randomly genotyped bull calves. For scenario GPROG_BD_BLUP, a preselection step is introduced. Bull dams are preselected according to their conventional BLUP EBV and only their male offspring has to be genotyped. Scenario GPROG_BD_PHEN is similar to scenario GPROG_BD_BLUP, but the preselection step is based on phenotypes of cows. In scenario GPROG_BD_HERD, selection of bull dams is restricted to specific herds that could be called "supplier herds" (Weigel, 2008) because they are the source to supply new genetic material. Scenario GPROG_ BD_HERD can also be viewed as the initiation of a nucleus scheme, where herds are selected to form the nucleus, and from then on selection, at least of females as future bull dams, is practiced within these herds. As explained above, large herds of 500 were formed to simulate this situation. To avoid a further preselection step, which would again create a need for variation, all females in these herds were considered as potential bull dams and hence selection took place within their male calves. Finally, in scenario GPROG_BD_GENO, all animals involved in the selection process-bull sires, bull dams, and their male offspring - will have a GBV. Preselection steps in GPROG_BD_GENO hence are based on the GBV of parents. For all scenarios GPROG_RANDOM to GPROG_BD_GENO, the number of bull calves or bull dams with either 0.5 or 1 male offspring, was varied by increments of 1,000 from 1,000 to 5,000 bull calves with genotypes, and hence with GBV, available for selection. A variation of the reproductive rate for bull dams is not reasonable for all scenarios. This pertains to GPROG_ALL and GPROG_RANDOM and hence was not simulated.

In summary, all scenarios (REF, GPAR, and GPROG) can also be viewed as young bull programs in which young bulls enter service directly as AI bulls. The question of acceptance of this genetic material by breeders and commercial dairy producers is not ad- dressed but has been discussed elsewhere (e.g., König et al., 2009). Neglecting the specific topic of the reproductive rate of bull dams, 10 main scenarios were compared. Accounting for the variations of preselected animals in all scenarios except REF, GPAR_ALL, and GPROG_ALL, 34 scenarios were evaluated for 6 levels of $r_{m g}$, and the entire set was repeated for heritabilities of 0.10 and 0.30 .

\section{Criteria for Comparison}

Breeding organizations will most likely seek to compare alternative selection strategies based on the average of breeding values of the bulls finally selected for AI because this parameter will determine their share in a competitive market. Scenarios that will be optimal under this criterion will also be the scenarios resulting in maximization of genetic gain. Averages and SD of TBV for varying numbers of selected animals were used. This is also in line with studies by de Roos et al. (2010) and Winkelman and Spelman (2010). Given that genetic evaluations performed will be unbiased, animals selected based on TBV as available in simulation studies will be identical to animals selected based on EBV with high accuracy.

An evaluation of alternate breeding strategy would not be complete without examining inbreeding coefficients of the selected animals, because only strategies with acceptable inbreeding coefficients will be sustainable. This is acknowledged by most authors conducting similar simulation studies (e.g., Pryce et al., 2010a,b). Inbreeding coefficients were analyzed as supplied for all individual animals by the QMSim program.

\section{RESULTS AND DISCUSSION}

Results for a comparison of all 10 main scenarios, including all sub-scenarios for preselection steps, are given in Figure 2 for a heritability $\left(h^{2}\right)$ of 0.10 and an accuracy $\left(r_{m g}\right)$ of 0.70 and in Figure 3 for a $h^{2}$ of 0.30 and an $r_{m g}$ of 0.90 . These 2 sets of parameters were chosen because they reflect the German situation for genomic selection in Holsteins based on the EuroGenomics calibration sample. For functional traits with a low heritability, $r_{m g}$ around 0.70 are achieved and for traits with a moderate heritability of around $0.30, r_{m g}$ close to 0.90 have been obtained. All values on the $y-$ axis of the 2 graphs refer to the average of the TBV. Under the parameters used to simulate the population, the SD of TBV is 0.32 for $h^{2}=0.10$ and 0.55 for $h^{2}$ $=0.30$. Scenario REF denotes the reference scheme under which bull calves are selected according to their pedigree index. It is known that a widespread use of 


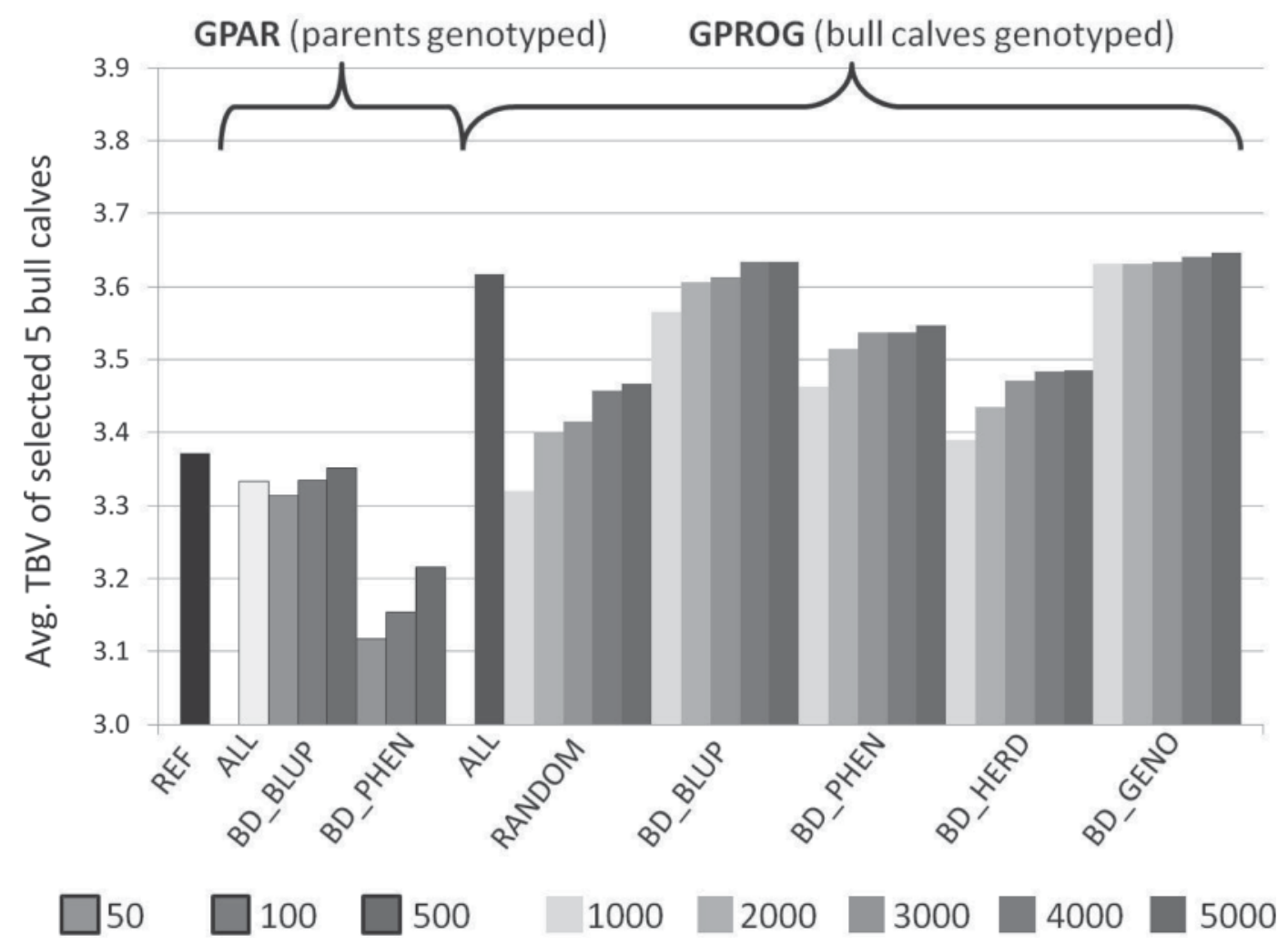

Figure 2. Average of true breeding values (TBV) of 5 selected bull calves for the scenarios examined under parameters $h^{2}=0.1$ and accuracy of the genomic breeding value $\left(r_{m g}\right)=0.7$. Bars showing 50,100, and 500 represent the number of preselected dams; 1000 to 5000 represent the number of bull calves genotyped. See Figure 1 for explanation of scenarios.

young bulls would be advantageous, as recently shown by Buch et al. (2010). For all scenarios, the averages of the TBV of the best 5 bulls are given.

In GPAR scenarios, the bull calves are not genotyped. This leads to a substantial disadvantage compared with GPROG scenarios and reflects the fact that an additional meiosis is involved from parents to sons. Generalizing, this disadvantage will amount to $1 \mathrm{SD}$ of TBV between the best GPAR and GPROG scenarios. In scenario GPAR_ALL, there is no shortage in potential bull dams as all females are genotyped and sires of bulls are always genotyped. Selection of bull calves under GPAR_ALL is based on genomic pedigree index. Especially for a low $r_{m g}=0.70$, improvements can be obtained when a preselection step is implemented involving 50, 100, and especially 500 bull dams preselected based on their conventional BLUP EBV. This finding may be surprising but is readily explained by the fact that for low $r_{m g}$, the access to a large population of genotyped females is not very helpful when the goal is to find superior animals according to their TBV. As expected, a preselection of bull dams according to phenotype results in the lowest average values of TBV of bull calves. This is especially evident for low heritability (Figure 2). Thus, breeding organizations or individual marketers of genetic material without access to the technology of genomic selection will not be competitive when attempting to market young bulls or their semen based on GBV of their parents only. For moderate heritability (Figure 3), however, this low-cost strategy would be competitive to the reference scenario. This strategy could then be called an improved juvenile scheme.

All GPROG scenarios obtain impressive results of an advantage of around 1.0 SD (Figure 2) or 1.2 SD (Figure 3 ) over the reference scenario. Scenario GPROG_ALL refers to the very idealistic scenario under which all male calves would be genotyped. However, as can be seen when comparing Figures 2 and 3, this would only be a distinct advantage for a higher $r_{m g}$. When comparing all scenarios, it should be kept in mind that Figures 2 and 3 display the average TBV of the 5 young bulls selected by their GBV. This explains why even an ideal situation of a completely genotyped male population will not be of great help when $r_{m g}$ is low and hence selection according to GBV is not accurate. As expected, a restriction of the pool of genotyped males available leads to lower values of TBV (scenario GPROG_RANDOM). However, the decrease is not as marked as could be envisaged and underlines that a "bull calf program" 


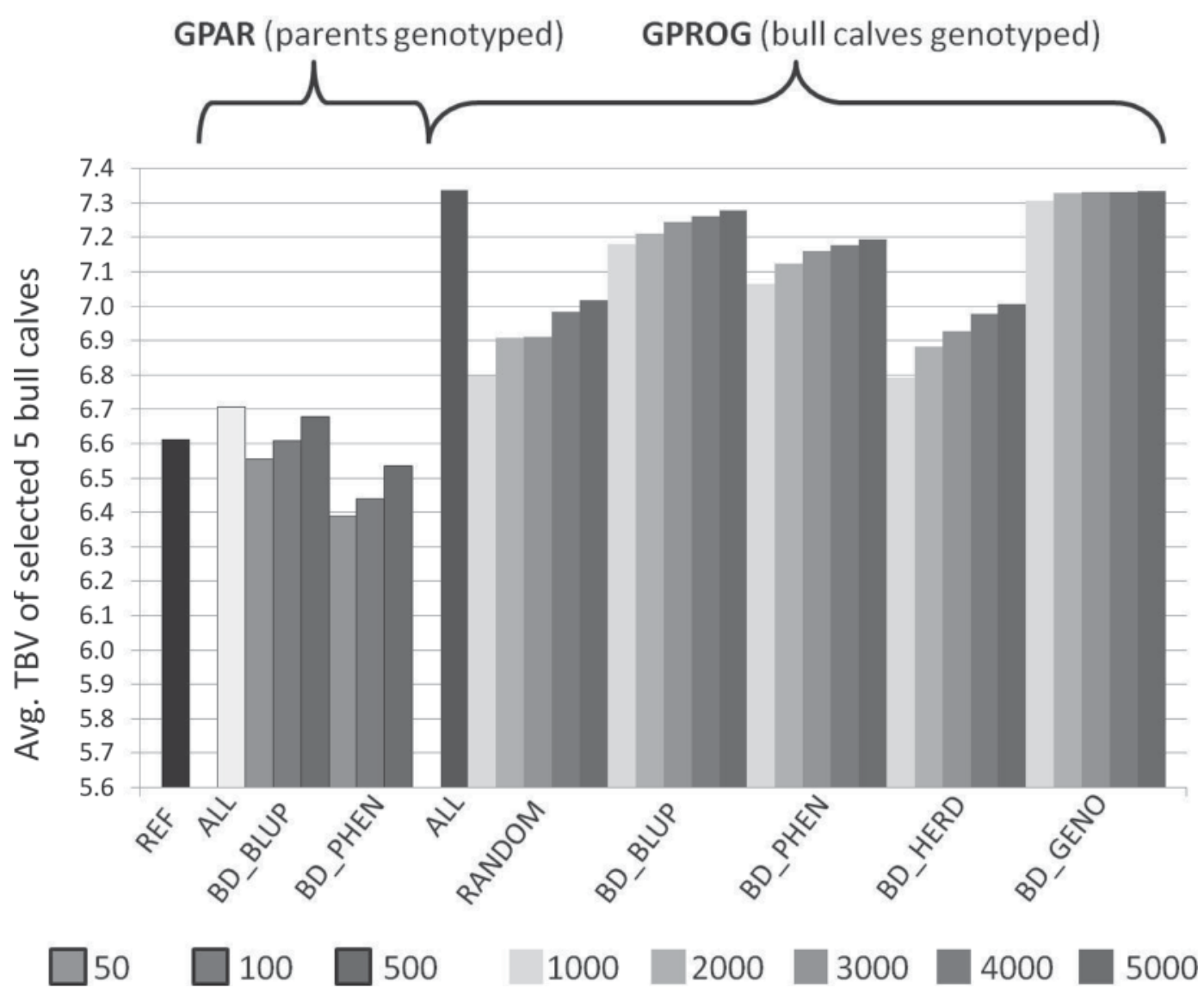

Figure 3. Average of true breeding values (TBV) of 5 selected bull calves for the scenarios examined under parameters $h^{2}=0.3$ and accuracy of the genomic breeding value $\left(r_{m g}\right)=0.9$. Bars showing 50,100, and 500 represent the number of preselected dams; 1000 to 5000 represent the number of bull calves genotyped. See Figure 1 for explanation of scenarios.

according to König and Swalve (2009) would indeed produce young bulls with superior breeding values compared with scenarios REF and GPAR. Even under a restriction of selecting from a pool of a few thousand genotyped bull calves only and completely neglecting any preselection, GPROG_RANDOM would be competitive with any REF or GPAR scenario. The comparison between scenarios GPAR_ALL and GPROG_ALL also can be interpreted as what is gained from genotyping male calves; that is, directly focusing on the product of a breeding organization, as opposed to concentrating on identifying superior females. In both scenarios, there is no shortage of genotyping; however, the superiority of GPROG_ALL in terms of the accuracy of selection is very clear.

A highly competitive scenario would be to include a preselection step for the bull dams and genotype their male offspring. Under low $r_{m g}$ (Figure 2), this strategy would be superior even to the idealistic scenario GPROG_ALL. For higher $r_{m g}$ (Figure 3), the advantage of GPROG_ALL over the GPROG_BD_BLUP scenarios would be small. Scenario GPROG_BD_
BLUP would also be cost-effective, because bull dams are not required to have genotype information. Thus, investments could be concentrated on genotyping an increasing number of bull calves from bull dams that are preselected according to their conventional BLUP EBV. Increasing the number of bull calves to be genotyped will be very beneficial for low $r_{m g}$ and an increase from 1,000 to 2,000 bull calves (Figure 2), but benefits will be small or negligible thereafter. For higher $r_{m g}$ and heritability (Figure 3), a preselection step will still yield improvements.

Analogous to the comparison of GPAR scenarios, for GPROG scenarios a preselection step for bull dams based on phenotypes is also not advisable. Again and as expected, the differences between scenarios GPROG BD_BLUP and GPROG_BD_PHEN are larger for a low heritability (Figure 2) compared with a moderate heritability (Figure 3). An even stronger reduction in the average TBV of the 5 selected bull calves is obtained when selection is practiced in a few herds that have been preselected based on herd effects (scenario GPROG_BD_HERD). This result would underline 
that when selection is going to be conducted in selected herds only, these herds should be preselected according to genetic merit.

Highest averages of TBV of selected bull calves can be achieved when bull dams in addition to bull sires are preselected based on their GBV and additionally bull calves will have GBV (scenario GPROG_BD_GENO). However, as is evident when comparing GPROG_ BD_BLUP and GPROG_BD_GENO, the advantage of the latter over the former would not be large. The slight increase has to be paid for through the costs of genotyping all potential bull dams. In practice, some scheme in between GPROG_BD_BLUP and GPROG_ BD_GENO should be sought as many bull dams will be genotyped at the expense of the breeders and thus the breeding organization could follow a strategy of not genotyping bull dams at their own expense. This result is in line with Sorensen and Sorensen (2010), who stated that it would not be advisable to genotype females unless operating under a multiple-ovulation embryo transfer (MOET) situation with 5 offspring per female. As explained above, in the present study, a "limited MOET condition" was assumed with 1 male offspring per selected female.

The question on whether to genotype females as potential bull dams also relates to the question of the extent and effect of preferential treatment of potential bull dams in dairy cattle breeding programs. A distinct advantage of genotyping females and basing any selection on GBV instead of conventional ones is that genomic selection nullifies the problem of preferential treatment (König and Swalve, 2009). Therefore, the ultimate decision of a breeding organization on whether to genotype females and pay for the genotyping should be based on the extent to which identifying superior females by conventional means is disturbed by preferential treatment. If a substantial extent of preferential treatment has to be assumed, scenario GPROG_BD_ GENO would be the scheme of choice.

Figures 4 and 5 contain details on the mean value of TBV for all GPROG scenarios, because these scenarios are clearly superior over REF and GPAR as seen from Figures 2 and 3. Figures 4 and 5 allow for a comparison of different $r_{m g}$ (0.5 to 0.9$)$ for both values of $h^{2}: 0.10$ and 0.30 . In scenario GPROG_ALL, all bull calves in the population are assumed genotyped. In scenario GPROG_BD_GENO, GBV are available for bull sires, bull dams, and bull calves. Both scenarios perform equally, with increases in average TBV from $r_{m g}=0.5$ to $r_{m g}=0.9$, amounting to $1.5 \mathrm{SD}$ for $h^{2}=0.1$ and to 1.4 SD for $h^{2}=0.30$. These are substantial increases and explain the high variation of literature results based on different assumptions for $r_{m g}$. Results for a different value for the number of selected bull calves are not shown because no changes of relative differences between scenarios result from different values. Averages of TBV for higher numbers of selected bull calves simply decrease due to decreasing selection intensity. For a range of 5 to 20 selected bull calves, differences in average TBV are marginal.

Standard deviations of TBV (not shown in figures) were examined. As expected, SD increased with increasing heritability and increasing average TBV. In addition, SD for small numbers of selected bull calves were higher than those for larger numbers. This finding is due to the stochastic nature of the simulation and reflects random fluctuations, which are more visible when considering only very few selected animals.

Average inbreeding coefficients of selected bull calves for the GPROG scenarios, when varying $r_{m g}$ and $\backslash$ the number of selected bull calves, 5 or 20, were examined. Differences for inbreeding coefficients between the 2 values of the number of selected bull calves were very small. For $h^{2}=0.10$, average inbreeding coefficients were in the range of 6.86 to $8.40 \%$ and substantially higher than those for $h^{2}=0.30$ (4.80 to $\left.5.90 \%\right)$. A reason for this might be that the information from relatives becomes less important with increasing heritability. Within each set of results for $h^{2}=0.10$ or $h^{2}$ $=0.30$, scenario GPROG_BD_BLUP clearly resulted in higher levels of inbreeding because this scenario relies on preselection of bull dams according to conventional BLUP EBV. Average inbreeding coefficients for GPROG_BD_BLUP were in the range of 7.72 to $8.40 \%$ for $h^{2}=0.10$ and in the range of 5.26 to $5.90 \%$ for $h^{2}=0.30$.

Inbreeding coefficients of selected animals in one generation can be helpful information but may not be fully informative for an assessment of future inbreeding. As the entire simulation and selection of animals in the present study focuses on the very last generation, an additional hint on future inbreeding may be taken from the relationship of the selected animals. The number of sons per sire of sons was taken as an indicator value. This value was calculated based on determining the size of the half-sib group from which each selected calf would come and then averaged over the number of selected bull calves, 5 and 20, respectively. As the simulation scheme used is not optimized for a multi-path selection model, many sire of sons may be sires of selected bull calves. Hence, values close to unity indicate that all selected bull calves came from different sire families. In general, differences between all GPROG scenarios were marginal and in the range of 1.000 to 1.284 for $h^{2}=$ 0.1 and 1.000 to 1.277 for $h^{2}=0.30$. However, scenario GPROG_BD_GENO, under which sires of sons, bull dams and the young bull calves are genotyped, tends to favor fewer sires of sons and thus is an indication 


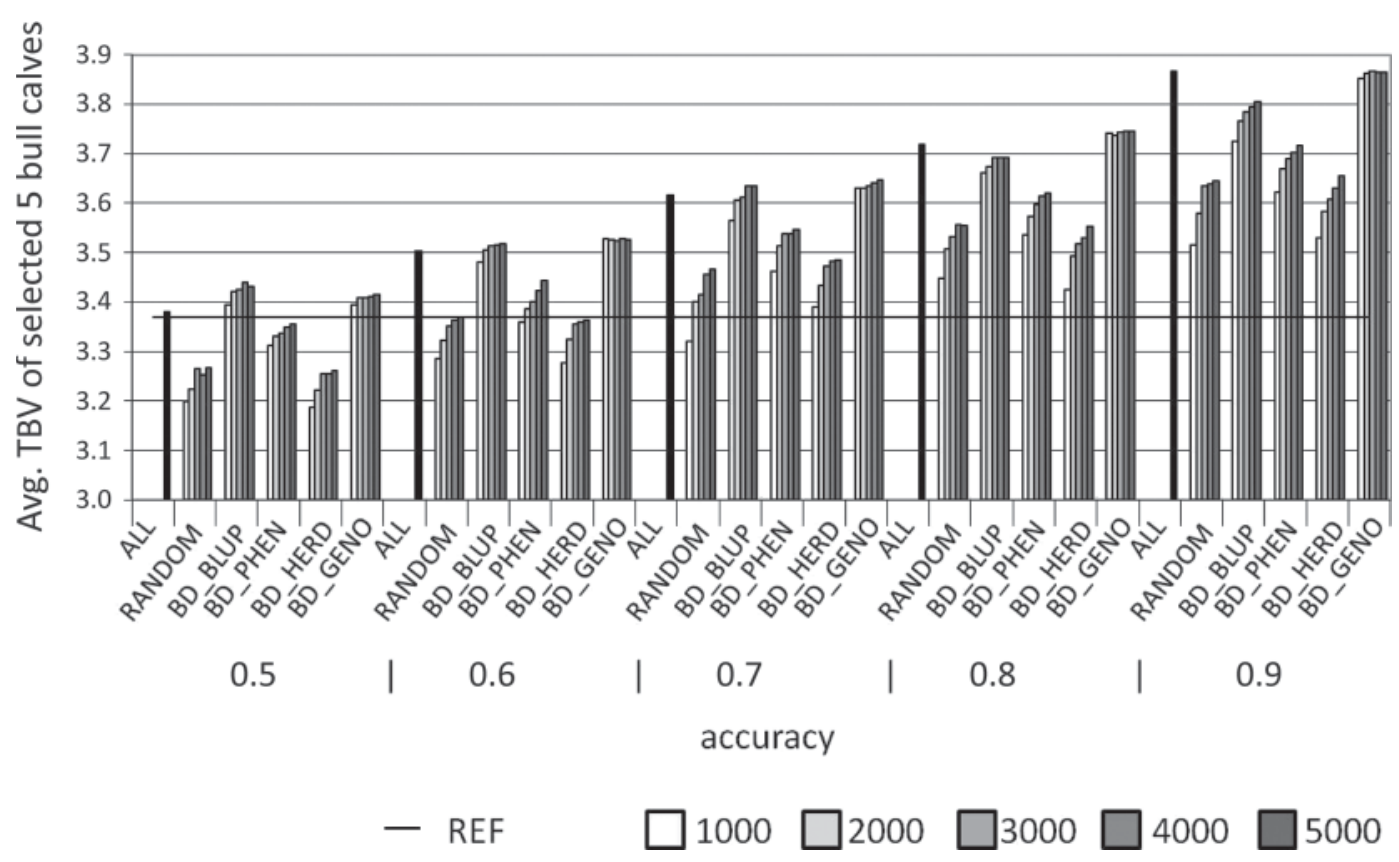

Figure 4. Average of true breeding values (TBV) of 5 selected bull calves for GPROG scenarios and the reference (REF) scenario under parameters $h^{2}=0.1$ and varying accuracy of the genomic breeding value $\left(r_{m g}\right)$ from 0.5 to 0.9 . Bars showing 1000 to 5000 represent the number of bull calves genotyped. See Figure 1 for explanation of scenarios.

for higher future inbreeding compared with all other scenarios. However, under scenario REF, the average size of the half-sib group for selected bull calves for $h^{2}$ $=0.10$ was 1.707 ( $\mathrm{n}=5$ selected bull calves) and 2.881 ( $\mathrm{n}=20$ selected bull calves) and the average size of the half-sib group for $h^{2}=0.30$ was $1.695(\mathrm{n}=5$ selected bull calves) and 2.579 ( $\mathrm{n}=20$ selected bull calves). Given that scenario REF can be viewed as the initial step of a conventional progeny-testing scheme, this is a very strong indication that breeding programs based

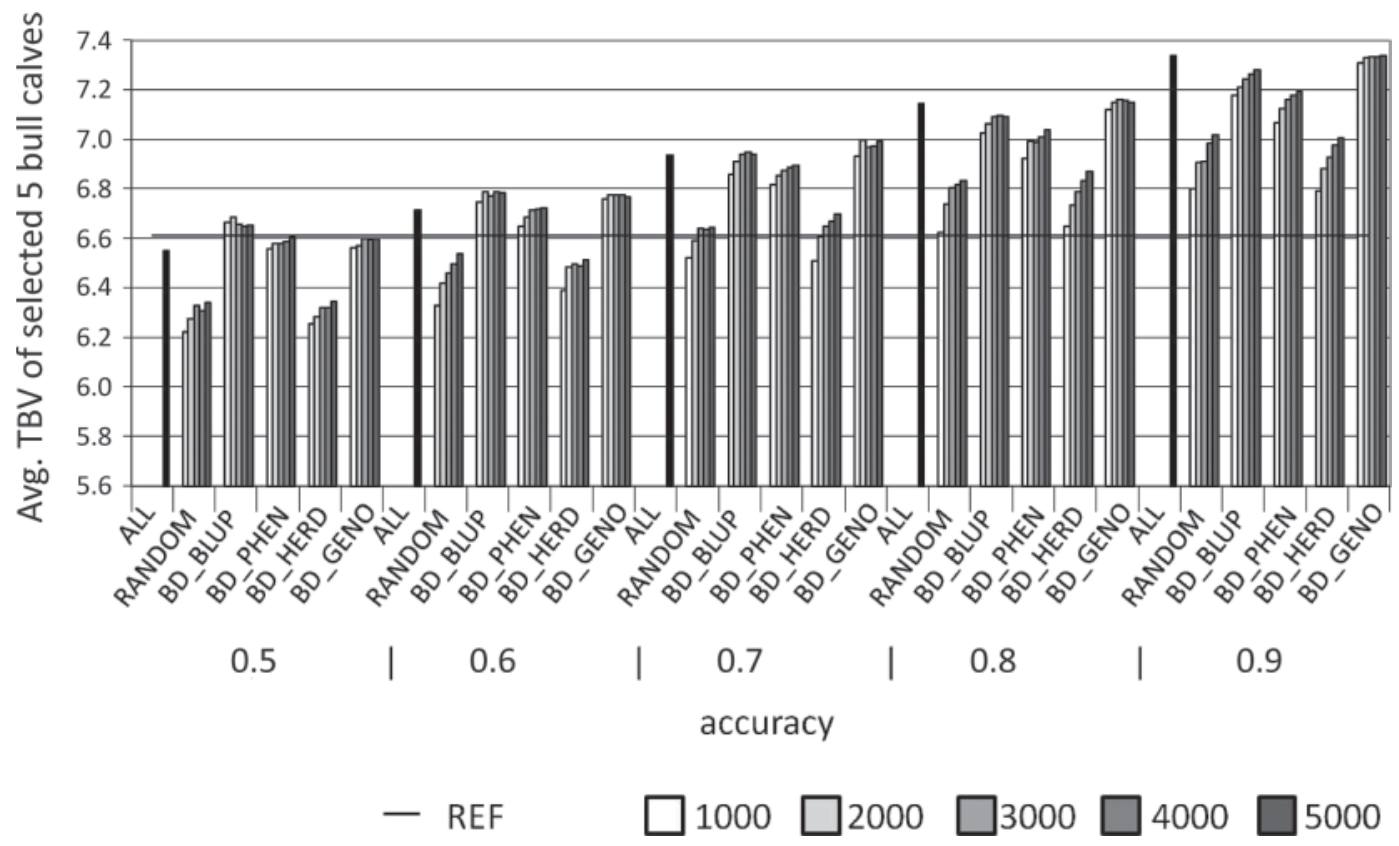

Figure 5. Average of true breeding values (TBV) of 5 selected bull calves for GPROG scenarios and the reference (REF) scenario under parameters $h^{2}=0.3$ and varying accuracy of the genomic breeding value $\left(r_{m g}\right)$ from 0.5 to 0.9 . Bars showing 1000 to 5000 represent the number of bull calves genotyped. See Figure 1 for explanation of scenarios. 
on genomic breeding values lead to lower inbreeding compared with conventional programs, which was also underlined by de Roos et al. (2010).

In their deterministic model calculation, König et al. (2009) examined the competitiveness of genomic selection relative to a conventional progeny-testing program and found both approaches equal with respect to discounted profit when $r_{m g}$ was 0.40 . With increasing values of $r_{m g}$, the application of genomic selection was advantageous. Most studies trying to assess the benefits of genomic selection so far have worked with fixed values for accuracies of genomic breeding values. Schaeffer (2006) used $r_{m g}=0.75$, and a very similar value of 0.71 was assumed in the studies of Buch et al. (2010), Sorensen and Sorensen (2010), and Pedersen et al. (2010). Relatively similar values for $r_{m g}^{2}$ of 0.44 and 0.52 in a 2 -stage selection procedure were used in the deterministic calculation of Winkelman and Spelman (2010). These values may appear somewhat low compared with values from large calibration samples as cited above but reflect the specific situation of the implementation of genomic selection in New Zealand. Apart from König et al. (2009), only the studies of de Roos et al. (2010) and especially Lillehammer et al. (2010) examined varying levels for $r_{m g}^{2}$. The latter study is, until now, the only simulation study to examine the benefits of genomic selection in a breeding program that analyzed a population across several generations accounting for the need of new calibration and considering the respective accuracies. Lillehammer et al. (2010) found a superiority of $46 \%$ of genomic selection with respect to genetic gain over a conventional progeny-testing breeding program. Lower values for the superiority of genomic selection over conventional programs were found only by Winkelman and Spelman (2010). This is in sharp contrast to other studies that have found an increase of genetic gain of $100 \%$ or even larger (e.g., Schaeffer, 2006; König et al., 2009; Buch et al., 2010). In the present study, for a heritability of $h^{2}=0.10$, all GPROG scenarios were on a level for the average TBV of selected bull calves, which is very similar to scenario REF when $r_{m g}$ is as low as 0.50 ; that is, the reliability of genomic breeding value is $25 \%$. Increases in $r_{m g}$ then lead to distinct numeric advantages up to around $12 \%$. For a higher heritability of $h^{2}=0.30$, again all GPROG scenarios are on an equal level with scenario REF for $r_{m g}=0.50$. Furthermore, the combination of $r_{m g}=0.50$ for $h^{2}=0.30$ appears to be quite far off from what would be expected even for small calibration samples. Increasing $r_{m g}$ to values of 0.70 or even higher results in advantages of GPROG scenarios of around 10.5\%. Hence, genomic breeding programs have the capacity to increase the average of the TBV of selected bull calves by around 10 to $12 \%$ compared with a young bull program. The reason for the varying degree of superiority of genomic selection when compared with other programs in the literature is presumably a result of the different assumptions on which different studies were based. This applies not only to the accuracy of genomic selection but also, to a large extent, on the selection intensities on the paths of selection that were studied. Unfortunately, virtually all studies mentioned so far have failed to analyze a wide range of selection intensities for various paths of selection. Most often, only fixed values were assumed. Examples are Schaeffer (2006) with a fixed value of 1,000 preselected bull dams and a fixed selection of 20 bull calves out of 500 bull dams, and Buch et al. (2010), who examined fixed values of 1,000 preselected males and 2,000 preselected females. Further examples include the work of Lillehammer et al. (2010), who used a fixed value of 750 genotyped males as a pool for selection, and Winkelman and Spelman (2010), when analyzing only 5 different options of selection intensities and strategies. Pryce et al. (2010b) examined the effect of varying the number of selection candidates to be genotyped for a range of 1,000 to 10,000 for scenarios comparable to the GPROG scenarios of the present study. An important result from their study was that increases in response to selection were very small for values of candidates larger than 5,000. This is very much in line with the present study, as further increases of average TBV beyond 5,000 candidates were not expected from the already marginal increases when comparing 4,000 to 5,000 candidates. It should be emphasized that scenario GPROG_ALL, denoting the unlimited availability of genotypes for young male candidates for selection only, is advantageous for medium to high reliability of GBV. For low reliability (i.e., <0.50), an unlimited availability of genotypes by itself is not helpful with respect to average TBV unless it is combined with a preselection of bull dams. In the case of preselection of bull dams, as simulated in scenarios GPROG_BD_BLUP and GPROG_BD_GENO, then an "unlimited" availability can be reduced to values below 5,000 candidates.

The examination of a wide range of selection intensities and selection strategies is of great interest to breeding organizations. This is because the number of animals to be genotyped from the point of view of a breeding organization is largely a function of costs. The costs of genotyping with high-density SNP arrays have already seen a substantial decrease compared with the early days of genomic selection and vary with the density of arrays. Low-density arrays with 3,000 to 4,000 SNP may have to be taken into account, at least for preselection steps (Weigel et al., 2010), and early highdensity arrays such as the Illumina 50K chip (Illumina, 
San Diego, CA) are now replaced or accompanied by arrays of much higher density.

Selection intensities, however, are not the only parameters that need variation in simulation studies. Intensity will be interwoven with strategy. A good example in this respect is whether it is worthwhile to genotype females in addition to male animals. Hayes et al. (2009) stress this point and refer to Schaeffer (2006), who concluded that genotyping females could have a higher contribution to genetic gain than genotyping males. However, it may be argued that this statement is not independent of the assumption of fixed selection intensities when preselecting bull dams and selecting bull calves from these dams. Spelman et al. (2010) reported on the practical experience of implementing genomic selection in New Zealand. These authors concluded that genotyping of females had been abolished completely by the breeding organization because it was not cost effective. König and Swalve (2009) argued that under the assumption of low costs for genotyping, a breeding program could be envisaged consisting of genotyping as many bull calves as possible and even ignoring any preselection. Such a breeding program would narrow almost all selection decisions into one path of selection and would be competitive with a Schaeffer-type genomic selection program when assuming selection fractions of $<0.1 \%$. Clearly, an application of such a program would only be possible for very low cost genotyping and could be improved upon by any reasonable preselection of dams of bulls. From our results, the main conclusion is that bull calves should always be genotyped. Scenario GPROG_BD_BLUP reaches response parameters on a level comparable to that of GPROG_BD_GENO or GPROG_ALL without having to genotype females. For specific sets of parameters $\left(h^{2}\right.$, $r_{m g}$ ), it could be advantageous to promote genotyping of females in such a way that this would result in a GPROG_BD_GENO strategy; that is, assuming that a large number of females would have been genotyped. A GPROG_BD_GENO strategy would take advantage of the fact that many breeders would genotype their best cows at their own expense.

The question of whether to genotype females is also not independent of the reproductive rate assumed for females. This was shown by Sorensen and Sorensen (2010), who assumed either 1 offspring per dam or 5 in a MOET situation. Under MOET, it was clearly beneficial to genotype females. Similar results were obtained by Pryce et al. (2010b). In the present study, a more pessimistic approach toward the success rates possible under embryo transfer was taken. One male offspring per female was assumed and a decreased rate mimicking a situation without embryo transfer was examined. This latter scenario affected the results for the
REF scenario. Relative differences between the 2 sets of results for GPAR and GPROG scenarios, however, were too marginal to warrant presentation of results and did not result in a change of the relative superiority of scenarios.

\section{CONCLUSIONS}

Breeding programs for dairy cattle that are partly or completely based on breeding values estimated using genomic information and neglecting phenotypes for young selection candidates have the potential of at least doubling the selection response. In comparison with idealistic scenarios under which all available male calves are genotyped, a very similar selection response can be achieved by genotyping only a few thousand selection candidates; that is, bull calves, if bull dams are preselected based on conventional EBV or on GBV and assuming that all sires of future sons are genotyped. A restriction of the selection of bull calves based on their GBV to herds selected by phenotypic herd average is not advisable. In general, phenotypic information should not be used for preselection of animals to be genotyped. Preselection steps based on conventional EBV always outperform the use of phenotypic information. The need for a sufficient accuracy of genomic breeding values was highlighted by the results of the present study. Inbreeding can be reduced when using GBV and hence such breeding programs will lead to an increase of sustainability with respect to genetic diversity.

\section{ACKNOWLEDGMENTS}

The authors are indebted to the German Holstein Association (DHV, Bonn, Germany) and its member organizations that supported the project with a grant.

\section{REFERENCES}

Buch, L. H., M. K. Sorensen, J. Lassen, P. Berg, and A. C. Sorensen. 2010. Dairy cattle breeding schemes with or without genomic selection and progeny testing. Abstract 418 in Proc. 9th World Congr. Genet. Appl. Livest. Prod., Leipzig, Germany. German Society for Animal Science, Leipzig, Germany.

de Roos, A. P. W., C. Schrooten, R. F. Veerkamp, and J. A. M. van Arendonk. 2010. The impact of genomic selection and short generation interval on dairy cattle breeding programs. Abstract 706 in Proc. 9th World Congr. Genet. Appl. Livest. Prod., Leipzig, Germany. German Society for Animal Science, Leipzig, Germany.

Goddard, M. E., and B. J. Hayes. 2009. Mapping genes for complex traits in domestic animals and their use in breeding programmes. Nat. Rev. Genet. 10:381-391.

Hayes, B. J., P. J. Bowman, A. J. Chamberlain, and M. E. Goddard. 2009. Invited review: Genomic selection in dairy cattle: Progress and challenges. J. Dairy Sci. 92:433-443.

Interbull. 2010. Accessed November 21, 2010. http://www.interbull. org/index.php?option=com_content\&view $=$ article\&id $=82 \&$ Item id $=119$. 
König, S., H. Simianer, and A. Willam. 2009. Economic evaluation of genomic breeding programs. J. Dairy Sci. 92:382-391.

König, S., and H. H. Swalve. 2009. Application of selection index calculations to determine selection strategies in genomic breeding programs. J. Dairy Sci. 92:5292-5303.

Lillehammer, M., T. H. E. Meuwissen, and A. K. Sonesson. 2010. Effects of alternative genomic selection schemes on genetic gain in dairy cattle. Abstract 130 in Proc. 9th World Congr. Genet. Appl. Livest. Prod., Leipzig, Germany. German Society for Animal Science, Leipzig, Germany.

Liu, Z., F. Seefried, F. Reinhardt, G. Thaller, and R. Reents. 2010 Dairy cattle genetic evaluation enhanced with genomic selection. Abstract 9 in Proc. 9th World Congr. Genet. Appl. Livest. Prod., Leipzig, Germany. German Society for Animal Science, Leipzig, Germany.

Lund, M. S., A. P. W. de Roos, A. G. de Vries, T. Druet, V. Ducrocq, S. Fritz, F. Guillaume, B. Gulbrandtsen, Z. Liu, R. Reents, C. Schrooten, M. Seefried, and G. Su. 2010. Improving genomic prediction by EuroGenomics collaboration. Abstract 880 in Proc. 9th Wld. Congr. Genet. Appl. Livest. Prod., Leipzig, Germany. German Society for Animal Science, Leipzig, Germany.

Meuwissen, T. H. E., B. Hayes, and M. E. Goddard. 2001. Prediction of total genetic value using genome-wide dense marker maps. Genetics 157:1819-1829.

Pedersen, L. D., M. K. Soresnsen, P. Berg, J. V. Andersen, and A. C. Sorensen. 2010. Using sexed semen has limited effect on genetic gain in a dairy cattle breeding scheme using genomic selection. Abstract 714 in Proc. 9th World Congr. Genet. Appl. Livest. Prod., Leipzig, Germany. German Society for Animal Science, Leipzig, Germany.

Pryce, J. E., M. E. Goddard, and B. J. Hayes. 2010a. Breeding schemes for dairy cows under genomic selection-What can we do? Abstract 291 in Proc. 9th World Congr. Genet. Appl. Livest. Prod., Leipzig, Germany. German Society for Animal Science, Leipzig, Germany.

Pryce, J. E., M. E. Goddard, H. W. Raadsma, and B. J. Hayes. 2010b. Deterministic models of breeding scheme designs that incorporate genomic selection. J. Dairy Sci. 93:5455-5466.
Sargolzaei, M., and F. S. Schenkel. 2009. QMSim: A large-scale genome simulator for livestock. Bioinformatics 25:680-681. doi:10.1093/ bioinformatics/btp045.

Schaeffer, L. R. 2006. Strategy for applying genome-wide selection in dairy cattle. J. Anim. Breed. Genet. 123:218-223.

Sorensen, A. C., and M. K. Sorensen. 2010. Genotyping both males and females is favorable in genomic dairy cattle breeding schemes. Abstract 720 in Proc. 9th World Congr. Genet. Appl. Livest. Prod., Leipzig, Germany. German Society for Animal Science, Leipzig, Germany.

Spelman, R. J., J. Arias, M. D. Keehan, V. Obolonkin, A. M. Winkelman, D. L. Johnson, and B. L. Harris. 2010. Application of genomic selection in the New Zealand dairy cattle industry. Abstract 311 in Proc. 9th World Congr. Genet. Appl. Livest. Prod., Leipzig, Germany. German Society for Animal Science, Leipzig, Germany.

VanRaden, P. M., C. P. Van Tassel, G. R. Wiggans, T. S. Sonstegard, R. D. Schnabel, J. F. Taylor, and F. S. Schenkel. 2009. Invited review: Reliability of genomic predictions for North American Holstein bulls. J. Dairy Sci. 92:16-24.

Weigel, K. A. 2008. North American perspective on developments in performance testing of dairy cattle and applications in breeding programs. Book of Abstracts No. 14: 210 in Proc. 59th Ann. Mtg. EAAP, Vilnius, Lithuania. EAAP, Rome, Italy.

Weigel, K. A., G. de los Campos, A. I. Vazquez, C. P. Van Tassell, G. J. M. Rosa, D. Gianola, J. R. O'Connell, P. M. VanRaden, and G. R. Wiggans. 2010. Genomic selection and its effects on dairy cattle breeding programs. Abstract 119 in Proc. 9th World Congr. Genet. Appl. Livest. Prod., Leipzig, Germany. German Society for Animal Science, Leipzig, Germany.

Wiggans, G. R., T. A. Cooper, P. M. VanRaden, and M. V. Silva. 2010. Increased reliability of genetic evaluations for dairy cattle in the United States from use of genomic information. Abstract 476 in Proc. 9th World Congr. Genet. Appl. Livest. Prod., Leipzig, Germany. German Society for Animal Science, Leipzig, Germany.

Winkelman, A. M., and R. J. Spelman. 2010. Response using genomewide selection in dairy cattle breeding schemes. Abstract 290 in Proc. 9th World Congr. Genet. Appl. Livest. Prod., Leipzig, Germany. German Society for Animal Science, Leipzig, Germany. 